\title{
LIGHT ELEMENTS AS PROBES OF STELLAR INTERIORS
}

\author{
A. Baglin and P.-J. Morel \\ Observatoire de Nice \\ B.P. $\mathrm{n}^{\circ} 139$ \\ 06003 NICE Cedex \\ France
}

For stars cooler than $6400^{\circ} \mathrm{K}$, the lithium is observed to be depleted with respect to the universal value for Population I stars. The depletion depends on mass and age and occurs, both during the mainsequence (Hyades) and the pre-main-sequence (Pleiades).

The physical model of this depletion is the following: light elements are nuclearly destroyed at low temperature $\left(2 \sim 5 \times 10^{6}{ }^{\circ} \mathrm{K}\right)$ i.e. close to the star surface. The surface convective zone, eventually somehow extended by overshooting, plays the role of a fully mixed reservoir (FMR) of light elements. For $\mathrm{T}_{\text {eff }} \lesssim 5000^{\circ} \mathrm{K}$ this FMR embodies the nuclear destruction region (NDR) and the light elements are destroyed. For $\mathrm{T}_{\text {eff }}>5000^{\circ} \mathrm{K}$ the depletion is due to some transport process acting between the bottom of the FMR and the NDR (fig. 1). The observed abundances of light elements hence, are indicators of the nature of the transport process at work between the photosphere and the NDR.

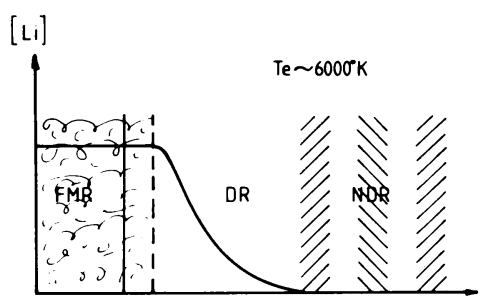

Using envelop models computed with the available physical data, Baglin et al. (1985) referred hereafter as BMS - showed that the depletion of lithium in the Hyades as observed by Cayrel et a1. (1983), was consistent with a turbulent difussion coefficient derived from the instability

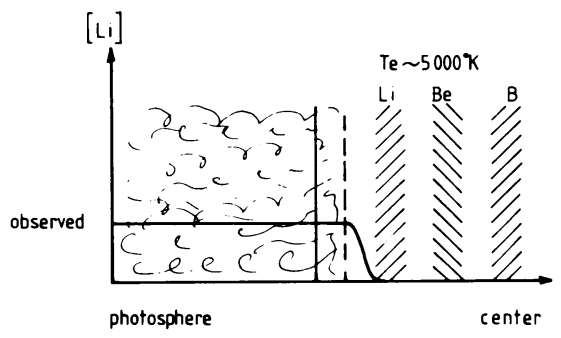

Figure 1. Scheme of the physical process.

G. Cayrel de Strobel and M. Spite (eds.), The Impact of Very High S/N Spectroscopy on Stellar Physics, 279-282.

(1) 1988 by the IAU. 
of the shear flow due to meridional circulation. However these results have to be revisited:

- New physical data are available, they allow to compute more reliable envelop models.

- The solar seismology has started to map the structure of the solar envelop, hence more sensitive observational tests are available.

Molecular opacity sources increase the opacity in the outerlayers and reduce the extend of the convective zone. This effect is larger for lower masses.

Heliosismology has shown that the bend of the temperature gradient of the solar convective zone occurs at $\sim 0.7 \mathrm{R}_{0}$. (Christensen-Dalsgaard et al., 1985). But solar envelopes computed with opacities including molecular sources, even with large mixing length have too shallow convective zones (Lebreton and Maeder, 1986).

The proposed modelization depends on two main free parameters:

$-\alpha=\ell / H_{p}$ the ratio of the mixing length to the pressure scale height.

- $\phi$ the "efficiency" of the diffusion process.

Different scenarios of the turbulent diffusion generation have been tested:

- Molecular analog (Schatzman, 1977):

$$
\mathrm{D}=\phi v
$$

- Shear of the meridional circulation (Zahn, 1984):

$$
\mathrm{D}=\phi \frac{\mathrm{r}}{\mathrm{g}\left(\nabla_{\mathrm{ad}}-\nabla_{\mathrm{rad}}\right)} \frac{\Omega^{2} \mathrm{R}^{2} \mathrm{~L}}{\mathrm{M}^{2}}
$$

- Simplified version (BMS, 1985):

$$
D=\frac{\phi}{\left(\nabla_{\text {ad }}-\nabla_{\text {rad }}\right)}
$$

- Saturation by dissipative process:

$$
\mathrm{D}=\phi=\text { cte }
$$

1- The Hyades sequence.

The lithium depletion for the Hyades sequences has been predicted as a function of $\alpha$ and $\phi$ (fig. 2). Errors bars, both in mass and abundance, define zone of solutions in the $(\alpha, \phi)$ place (fig. 3 ).

The scenario: "Saturation by dissipative process", with an overshooting of $0.23 \mathrm{H}_{\mathrm{p}}$, appears to be the only one for which the intersection of these zones is not empty. It corresponds to a value of the constant diffusion coefficient of $D \sim 2500$ and to a mixing length coefficient of $\alpha \sim 2.8$. With these values, the computed abundances are consistent with the observations (fig. 1). 


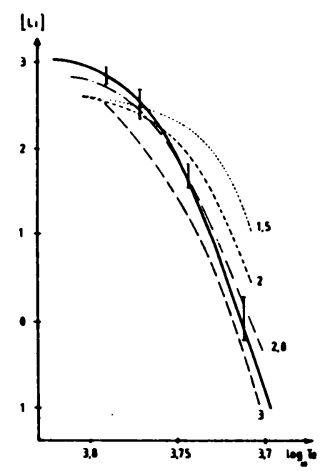

Figure 2. $|\mathrm{Li}|$ versus $\log _{10} \mathrm{~T}_{\text {eff }}$ for different values of $\alpha$ compared to the observations (vertical bars) The best fit is obtained for the bold curve which corresponds to $\alpha=2.8$, an overshooting region of . $23 \mathrm{H}_{\mathrm{p}}$ and $\mathrm{D}=2500$.

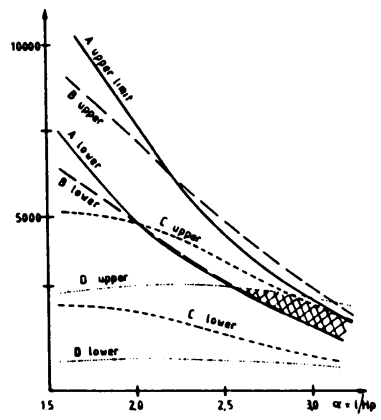

Figure 3. The $(\alpha, \Phi)$ plane for the scenario "Saturation by dissipative process". Models ABCD upper and lower limits correspond to the four observations shown in fig. 2 with their error bars.

\section{2- The Sun.}

The observed lithium abundance is: $|\mathrm{Li}|=0.96$. The computation with $\alpha=2.8$ and $D=2500=$ cte gives $|\mathrm{Li}|=-1.5$. If the mixing length ratio of 2.8 obtained for the Hyades is an universal value, a smaller $D=800$ is needed in the calculations to get the Sun lithium abundance of $|\mathrm{Li}|=0.96$. The observed beryllium abundance is $|\mathrm{Be}|=1.3$ (no depletion) and the computed value with $\alpha=2.8$ and $D=800$ is $|\mathrm{Be}|=0.87$ Since the beryllium is destroyed deeper than the lithium, the diffusion coefficient has to decrease with depth: the scenario " $D=$ cte" is not entirely consistent.

3- $\alpha$ Cen $A$ and $B$.

The relevant physical parameters for this visual binary are:

$$
\begin{aligned}
& \alpha \text { Cen } A: \mathrm{T}_{\text {eff }}=5780^{\circ} \mathrm{K}, \mathrm{M} / \mathrm{M}_{\Theta}=1.1, \mathrm{~L} / \mathrm{L}_{\Theta}=1.6,|\mathrm{Li}|=1.28 \\
& \alpha \operatorname{Cen} \mathrm{B}: \mathrm{T}_{\text {eff }}=5320^{\circ} \mathrm{K}, \mathrm{M} / \mathrm{M}_{\Theta}=0.9, \mathrm{~L} / \mathrm{L}_{\Theta}=.46,|\mathrm{Li}|=0.00
\end{aligned}
$$

The age is $\sim 4.25 \times 10^{9}$ years (Demarque et al., 1986). With $\alpha=2.8$ and $\mathrm{D}=2300=$ cte, one obtains results consistent with the observations: $|\mathrm{Li}|_{\mathrm{A}}=1.28$ and $|\mathrm{Li}|_{\mathrm{B}}=-7.7$.

\section{CONCLUSIONS.}

Light elements are powerful tools to probe stellar interior theory:

- Turbulent diffusion seems, up to now, the only mechanism which can reproduce the observed temperature dependence of lithium abundance in the Hyades.

- Convective zones seem to be deeper than actually predicted.

- The value of the diffusion coefficient obtained for $\alpha$ Cen is close 
to the Hyades value.

Improvements of the stellar interior theory need accurate abundance observations of light elements in homogeneous sets of stars like clusters or pairs of well known binaries.

\section{ACKNOWLEDGEMENTS .}

Yveline Lebreton, Roger Cayrel, Giusa Cayrel, Evry Schatzman, Nicolas Grevesse, Claude Burkhart.

\section{REFERENCES.}

Baglin, A., Morel, P.-J., Schatzman, E.: 1985, Astron. Astrophys. 149, 309.

Cayrel, R., Cayrel de Strobe1, G., Campbeil, B., Däppen, W.: 1984, Astrophys. J. 283, 205.

Christensen-Da1sgaard, J., Duva11, Jr, T.L., Gough, D.0., Harvey, J.W., Rhodes Jr, E.J.: 1985, Nature 315, 378.

Demarque, P., Guenter, D.B., van Altena, W.F.: 1986, Astrophys. J. 300, 773.

Lebreton, Y., Maeder, A.: 1986, Astron. Astrophys. 161, 119.

Schatzman, E.: 1977, Astron. Astrophys. 56, 211.

Zahn, J.-P.: 1984, in "Instabilités hydrodynamiques et applications

astrophysiques", p. 389, eds. A. Baglin et M. Auvergne, S.F.S.A. Paris.

\section{DISCUSSION}

BECKRAN A remark: In to morrow's session, Rebolo and I will present a study of $\mathrm{Li}$ depletion in the lower temperature part of the Hyades main sequence, i.e. for temperatures below 6300K. We have separated the effect of rotation from the effect of temperature, and show that at lower rotational velocity there appears to be less lithium. These observations offer us an additional means to probe the nature of the physical mechanism which drive the depletion, i.e. we can see how it depends on rotation for stars of equal temperature. 